\title{
Mitteilungen der Sektion
}

\section{Offene Sektionstagung im Oktober 2005}

Am 6. und 7. Oktober 2005 fand an der Universität Mannheim mit Unterstützung des Mannheimer Zentrums für Europäische Sozialforschung (MZES) die erste Offene Sektionstagung statt. In 36 Panels wurden vor ca. 230 Teilnehmerinnen und Teilnehmern 119 Papiere präsentiert. Davon können 76 nach wie vor unter http://www.mzes.unimannheim.de/konf/dvpw2005/fs_programm.html heruntergeladen werden.

\section{DVPW-Kongress}

Im Rahmen des Kongresses organisiert die Sektion die folgenden zwei Panels:

- Globalisierung und Reform (gemeinsam mit der Sektion Politik und Ökonomie), 26. September 2006, 14-17 Uhr;

- Reform der Außenpolitik, 27. September 2006, 14-16 Uhr.

Die Mitgliederversammlung findet am 27. September 2006 von 16:30 bis 17:30 Uhr statt. Die vorläufige Tagesordnung besteht in folgenden drei Punkten: 1. Bericht des Sprechergremiums; 2. Neuwahl des Sprechergremiums; 3. Sonstiges.

\section{Wahlaufruf}

Beim DVPW-Kongress im September 2006 in Münster müssen die drei Sprecherinnen und Sprecher der Sektion Internationale Beziehungen neu gewählt werden. Keiner der jetzigen Sprecher wird wieder kandidieren. Wir würden es sehr begrüßen, wenn sich zahlreiche Kolleginnen und Kollegen zu einer Kandidatur bereit erklären würden. Interessenbekundungen und Kandidatenvorschläge können per E-Mail an jedes der derzeitigen Vorstandsmitglieder geschickt werden. 
Für Rückfragen stehen die Sprecher der Sektion unter folgenden Adressen zur Verfügung:

Prof. Dr. Gunther Hellmann

Johann Wolfgang Goethe-Universität Frankfurt a. M.

Institut für Vergleichende Politikwissenschaft und internationale Beziehungen

Robert-Mayer-Straße 5, Fach 102

60054 Frankfurt a. M.

Tel.: 069/798-25191 oder -22667

E-Mail: g.hellmann@soz.uni-frankfurt.de

http://www.soz.uni-frankfurt.de/hellmann/start.htm

Prof. Dr. Frank Schimmelfennig

ETH Zentrum SEI

Center for Comparative and International Studies

Seilergraben 49 (SEI G 12)

CH-8092 Zürich

Tel.: 0041 44/6328062

E-Mail: frank.schimmelfennig@ep.gess.ethz.ch

http://www.cis.ethz.ch/

PD Dr. Peter Rudolf (Geschäftsführung 1.10.2005 - 30.9.2006)

Stiftung Wissenschaft und Politik

Ludwigkirchplatz 3-4

10719 Berlin

Tel.: 0 30/8 80 07-2 42

E-Mail: peter.rudolf@swp-berlin.org

http://www.swp-berlin.org/ 